\title{
Amygdalar and Prefrontal Pathways to the Lateral Hypothalamus Are Activated by a Learned Cue That Stimulates Eating
}

\author{
Gorica D. Petrovich, Peter C. Holland, and Michela Gallagher \\ Department of Psychological and Brain Sciences, Johns Hopkins University, Baltimore, Maryland 21218
}

\begin{abstract}
Experimental animals that are trained to associate a cue with food consumption when hunger prevails will subsequently consume a greater amount of food when that cue is presented under conditions of satiety. Previously, we showed that this phenomenon of conditioned potentiation of feeding is abolished by a neurotoxic lesion that encompasses the basolateral (BL), basomedial (BM), and lateral (LA) nuclei of the amygdala (AMY) and by disconnection of this region and lateral hypothalamus (LHA). Here, we combined immediateearly gene (IEG) and tract-tracing methods to map functional AMY-LHA circuitry that is engaged when potentiated feeding is produced by pavlovian conditioning. Sated rats were assessed for food consumption in the presence of a cue that was paired previously with food $(\mathrm{CS}+)$, or in the presence of another cue that was never paired with food (CS-), in two consecutive tests temporally arranged for activation of the effector IEGs Arc (activity-regulated cytoskeletal protein) and Homer 1a. We examined the selective induction of the IEGs by tests with CS + or CS - presentations in AMY neurons that project to LHA, as identified with the retrograde tracer FluoroGold. Using the same labeling methods, we also examined neurons in several other forebrain regions, including the prefrontal cortex and nucleus accumbens, that receive strong inputs from BL/BM/LA nuclei and, in turn, innervate the LHA. Our results indicate that a cue that has acquired the ability to promote eating in sated rats $(\mathrm{CS}+)$ strongly activates a functional network formed by direct pathways from the $\mathrm{BL} / \mathrm{BM}$ and orbitomedial prefrontal cortex to the LHA.
\end{abstract}

Key words: appetite; feeding; learning; pavlovian conditioning; satiation; network

\section{Introduction}

Great progress is being made in defining the mechanisms and mediators for energy balance and maintenance of body weight, including hypothalamic neuropeptides and related neural circuits that promote or suppress feeding (Schwartz et al., 2000; Swanson, 2000; Berthoud, 2002; Grill and Kaplan, 2002; Saper et al., 2002; Moran, 2004). In addition to integrating information relevant to internal energy supplies and demands, the behavioral regulation of feeding is subject to many external factors that can tip the balance in weight control (Rodin, 1981; Booth, 1989; Stroebele and De Castro, 2004). The causes of weight gain and obesity today represent a major public health concern, yet little is known about the neural circuitry that is involved in the motivational control of appetite, which can override the regulation of food consumption based on energy needs alone. Studies using functional imaging methods have suggested that a number of forebrain areas, including the amygdala (AMY), participate in the activation and maintenance of appetite in humans (Tataranni et

Received March 18, 2005; revised July 28, 2005; accepted Aug. 3, 2005.

This work was supported by National Institute of Mental Health Grants MH67252 (G.D.P.) and MH60179 (M.G. P.C.H.). We thank Dr. Paul F. Worley for providing Arc and H1a probe constructs and Drs. Jennifer Bizon and John Guzowski for help with the in situ hybridization technique.

Correspondence should be addressed to Gorica Petrovich, Department of Psychological and Brain Sciences, Johns Hopkins University, 3400 North Charles Street, Baltimore, MD 21218. E-mail: petrovic@jhu.edu.

D0I:10.1523/JNEUROSCI.2480-05.2005

Copyright $\odot 2005$ Society for Neuroscience $\quad$ 0270-6474/05/258295-08\$15.00/0 al., 1999; LaBar et al., 2001; Gottfried et al., 2003; Killgore et al., 2003; Kringelbach et al., 2003; Hinton et al., 2004).

The AMY is strongly connected to the hypothalamus (Petrovich et al., 2001). Notably, outputs from the basolateral (BL) and basomedial (BM) nuclei to the lateral hypothalamus (LHA) provide a route to feeding circuitry, as demonstrated recently with methods for transsynaptic retrograde tracing (DeFalco et al., 2001). This region of the amygdala is implicated in the control of food consumption by extrinsic cues (Holland et al., 2002; Petrovich et al., 2002). The phenomenon of potentiated feeding produced by pavlovian conditioning (Weingarten, 1983) is abolished in laboratory rats by a neurotoxic lesion of $\mathrm{BL}, \mathrm{BM}$, and LA (Holland et al., 2002) and by a lesion that disconnects these regions from LHA (Petrovich et al., 2002). The disconnection preparation interferes with functions that require AMY-LHA integration by either direct or indirect pathways. The BL and BM possess direct projections to the LHA and also form part of a forebrain network with many indirect pathways for gaining access to the hypothalamus that could include regions of prefrontal cortex (PFC), the nucleus accumbens (ACB), and central nucleus of the amygdala (CEA), target areas of BL/BM/LA output that, in turn, innervate the LHA (Swanson and Petrovich, 1998).

In the current study, we used functional anatomical methods to identify forebrain projection neurons to the LHA that are selectively activated by a learned cue that stimulates eating. Sated rats were assessed for food consumption in the presence of either 
a cue that was paired previously with food (CS+), or in the presence of another cue that was never paired with food (CS-), in two consecutive tests arranged to allow detection of CS-evoked expression of the immediate-early genes (IEGs) activityregulated cytoskeletal protein $(A r c)$ and Homer $1 a(H 1 a)$ which have a time-limited appearance in the nucleus of activated neurons (Vazdarjanova et al., 2002; Vazdarjanova and Guzowski, 2004). A confocal analysis revealed that a learned cue that stimulates eating activates a functional network formed by direct pathways from the $\mathrm{BL} / \mathrm{BM}$ and orbitomedial prefrontal cortex (omPFC) to the LHA.

\section{Materials and Methods}

Subjects. Experimentally naive, male Long-Evans rats (Charles River Laboratories, Raleigh, NC), weighing $\sim 300 \mathrm{~g}$ on arrival in the vivarium, were individually caged, maintained on a $12 \mathrm{~h}$ light/dark cycle, and given ad libitum access to food and water, except as otherwise noted. After 1 week of acclimation to vivarium conditions, during which time they were handled extensively, rats underwent behavioral training procedures. After training, rats were given injections of the retrograde tracer FluoroGold (FG; Fluorochrome, Denver, CO) into the lateral hypothalamus, and, after recovery, they received a final behavioral test.

Surgical methods (retrograde tracer injections). All surgeries were performed under aseptic conditions, using isoflurane gas for induction and maintenance of anesthesia and a stereotaxic frame (Kopf Instruments, Tujunga, CA). FluoroGold injections $(0.1 \mu \mathrm{l}$ of $4 \%$ solution in $0.9 \%$ saline) were made with a 30 gauge needle attached by a length of plastic tubing to a $10 \mu \mathrm{l}$ microsyringe (Hamilton, Reno, NV) mounted on a syringe pump (Sage Instruments, Boston, MA). The placement of injections was balanced, such that there were approximately equal numbers of rats with injections in the left or right lateral hypothalamus. The flat skull coordinates from bregma were as follows: anteroposterior, $-2.70 \mathrm{~mm}$; mediolateral, $\pm 1.40 \mathrm{~mm}$; and dorsoventral, $-8.90 \mathrm{~mm}$.

Apparatus. Six identical behavioral chambers $(30 \times 24 \times 30 \mathrm{~cm}$; Colbourn Instruments, Allentown, PA), each with a grid floor, aluminum top and sides, and a transparent Plexiglas back and front, were used for training and testing. The interior length of each chamber was reduced by positioning a transparent Plexiglas partition at an angle such that the length of the floor was reduced to $22 \mathrm{~cm}$, but the length at the top of the box remained unaltered. On the side wall opposite the Plexiglas partition, each chamber contained a recessed food cup $(3.2 \times 4.2 \mathrm{~cm})$, into which food pellets (45 mg; P. J. Noyes, Lancaster, $\mathrm{NH}$ ) were delivered. Dim background illumination was provided by two $25 \mathrm{~W}$ red bulbs, each placed $\sim 1.5 \mathrm{~m}$ from the test chambers. Masking noise $(60 \mathrm{~dB})$ was provided by ventilation fans located outside each box. A 10 s tone $(1500 \mathrm{~Hz}$, $70 \mathrm{~dB})$ and a $10 \mathrm{~s}$ white noise $(70 \mathrm{~dB})$ were used as CS + and CS - . For approximately one-half of the animals in each group, the tone served as the $\mathrm{CS}+$, whereas the white noise served as the CS+ for the remaining half. The unconditioned stimulus consisted of two $45 \mathrm{mg}$ food pellets (P. J. Noyes) delivered at the termination of the CS+. Video cameras attached to videocassette recorders were placed in the back of the test chambers to record behavior for a $10 \mathrm{~s}$ period before and during stimulus presentation. Stimulus presentation and videocassette recorders were controlled by LabView (National Instruments, Austin, TX) software run on Macintosh computers (Apple Computers, Cupertino, CA).

Behavioral procedures. Before behavioral training, rats were gradually reduced to $85 \%$ of their ad libitum weight. After a shaping procedure in which rats learned to eat from the food cup, rats received two sessions (one session per day) of CS-food pairing. Each $32 \mathrm{~min}$ session consisted of eight presentations of the $10 \mathrm{~s}$ CS + (tone or noise), immediately followed by delivery of two pellets into the food cup. In each of the next 1132 min discrimination-training sessions, the rats received two reinforced presentations of the CS+ intermixed with six nonreinforced presentations of the other auditory stimulus (CS-). After completion of conditioning, rats were given $14 \mathrm{~d}$ of ad libitum access to food in their home cage. The day after the training, rats received FG injections. After $13 \mathrm{~d}$ of recovery, rats received consumption tests in the test chambers: one with the CS+ and the other with $\mathrm{CS}-$. The tests were arranged

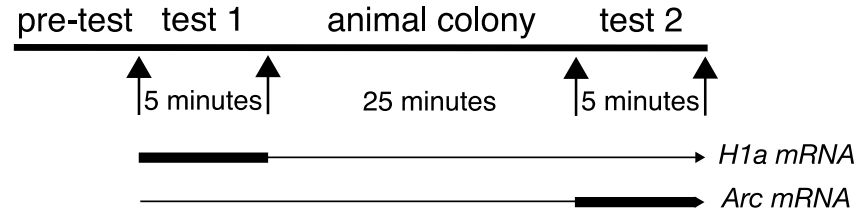

Figure 1. Experimental design. Sated rats were tested for food consumption in the presence of $\mathrm{CS}+$ or $\mathrm{CS}-$ in two consecutive tests (the order of tests was counterbalanced). The tests were arranged temporally to correlate with the peaks for nuclear mRNA induction of Arc and H1a. Each food-consumption test started with a pretest $(5 \mathrm{~min})$, immediately followed by test 1 ( $5 \mathrm{~min}$ ). After the first test, animals were taken to the colony and $25 \mathrm{~min}$ later were brought back for test 2 ( $5 \mathrm{~min})$. Immediately after the second test, rats were perfused. Thus, test 1 , which occurred $35-30$ min before the rats were killed, matches the peak of H1a nuclear mRNA induction, whereas test 2, which occurred 5 min before the rats were killed, matches the peak for $\operatorname{Arc}$ nuclear mRNA induction.

temporally for induction of nuclear mRNA for Arc (Lyford et al., 1995) [also known as Arg3.1 (Link et al., 1995)] and Hla (Brakeman et al., 1997), which have a time-limited appearance in the nucleus of activated neurons (Vazdarjanova et al., 2002; Vazdarjanova and Guzowski, 2004) (Fig. 1). Both the order of the tests (CS+ or CS-) and the identity of the CS (tone or noise) were counterbalanced. The protocol on a test day began with ad libitum access to food pellets in the home cage for $1 \mathrm{~h}$, immediately followed by a pretest and two tests in behavioral chambers. The $5 \mathrm{~min}$ pretest was included to provide a "baseline" for food consumption in the behavioral chamber and to reduce context-dependent consumption during the tests so that potentiation of eating by the CS+ would be readily apparent. For the pretest, rats were placed into the experimental chambers with 50 food pellets available in the food cup. At the completion of the pretest, the rats were removed from the chambers, the pellets remaining in the food cup were collected for counting, and the food cups were refilled with 50 new pellets. Rats were then promptly returned to the chambers for the first 5 min test. During that test, a total of $1010 \mathrm{~s} \mathrm{CS}+$ or CS - were presented. After the first test, the rats were returned to their home cage in the colony room for $25 \mathrm{~min}$ and then brought back for the second test. This test was identical to the first test, except that the other CS was presented. At the end of this test, the rats were removed, and the pellets remaining in the food cup were counted.

Histological procedure. Immediately after the end of the second foodconsumption test, the rats were deeply anesthetized with isoflurane and were intracardially perfused with $200 \mathrm{ml}$ of $0.9 \%$ saline, followed by 400 $\mathrm{ml}$ of $4 \%$ paraformaldehyde in $0.1 \mathrm{M}$ PBS. The brains were removed and stored overnight in the solution used for perfusion with $12 \%$ sucrose and then rapidly frozen with dry ice and kept at $-80^{\circ} \mathrm{C}$. The brains were sliced on a freezing microtome, and five series of $25-\mu \mathrm{m}$-thick coronal sections were collected into ice-cold $0.1 \mathrm{M}$ PBS. Immediately after cutting, a series intended for combined in situ hybridization and FG detection processing was mounted on slides (SuperPlus; Fisher Scientific, Pittsburgh, PA), vacuum dried overnight at room temperature, and then stored in an air-tight container with desiccant at $-80^{\circ} \mathrm{C}$. Another series of sections was processed for standard FG immunohistochemistry alone to ensure that the in situ hybridization treatment did not interfere with FG staining. A third series of adjacent sections was stained with thionin. The analysis of the FG pattern in tissue processed with FG alone was accomplished by using adjacent thionin-stained sections to demarcate nuclear borders (see below, Image acquisition and analysis). The brain areas were demarcated according to the Swanson (1998) atlas. The number of FG-labeled neurons was the same in tissue processed with FG alone compared with the number of FG-labeled neurons in the tissue processed with in situ hybridization. Thus, this series, which was stained with a blue/gray chromagen, Vector SG (Vector Laboratories, Burlingame, CA), provided us with a map of FG distribution that is easily visualized under light microscopy and that, together with adjacent thionin-stained sections, served as a guide for acquiring confocal z-stacks.

FG detection alone. FluoroGold was detected by a standard immunohistochemical procedure. In brief, free-floating sections were incubated with anti-FluoroGold antibody (Chemicon, Temecula, CA) in $0.1 \mathrm{~m} \mathrm{PBS,}$ $0.3 \%$ Triton $\mathrm{X}-100$, and $2 \% \mathrm{NGS}$ for $72 \mathrm{~h}$ at $4^{\circ} \mathrm{C}$, followed by incubation 
with biotinylated secondary antibody (anti-rabbit; Vector Laboratories), and avidin-biotin complex (Vector Laboratories), which was visualized with Vector SG (Vector Laboratories). After staining, tissue was mounted on SuperPlus slides (Fisher Scientific), dried, and coverslipped with DPX Mountant (Electron Microscopy Sciences, Hatfield, PA).

Combined double-label fluorescence in situ hybidization and FG detection. After the success of FG injections within the LHA was confirmed with FG immunohistochemistry alone in one set of sections, a separate set of sections from the same brains were processed with combined double-label fluorescence in situ hybridization (FISH) for Arc and H1a the nuclear, Nissl-type stain 4',6-diamidino-2-phenylindole (DAPI). Double-label FISH for the IEGs Arc and Homer $1 a$ was performed according to modified protocols of Vazdarjanova et al. (2002) and Simmons and Swanson (Simmons et al., 1989). Slides were pretreated with proteinase $\mathrm{K}$ in $5 \%$ SDS buffer and then treated with $0.5 \%$ acetic anhydride $1.5 \%$ triethanolamine, equilibrated in $2 \times$ SSC and dehydrated through serial ethanol solutions. Hybridization solution that contained cRNA probes (with incorporated digoxigenin-UTP for Arc and fluorescein-UTP for $\mathrm{H1a}$ ) was applied to the tissue, which was then hybridized for $20 \mathrm{~h}$ at $60^{\circ} \mathrm{C}$. A commercial transcription kit (MaxScript; Ambion, Austin, TX) and premixed RNA-labeling nucleotide mixes containing either digoxigenin or fluorescein-labeled UTP (Roche Applied Science, Indianapolis, IN) were used to generate cRNA riboprobes. Riboprobes were purified with EtOH precipitation, and the yield and integrity of the probes was determined by dot-blot analysis. The plasmid used for generating $A r c$ antisense and sense riboprobes contained fulllength cDNA ( $\sim 3.0 \mathrm{kbp}$ ) of the Arc transcript (Lyford et al., 1995). The antisense riboprobe for $H 1 a$ was directed to the $3.50 \mathrm{~kb} \mathrm{3} 3^{\prime}$-untranslated region (UTR) of the H1a mRNA (Brakeman et al., 1997). After hybridization slides were treated with RNase $(20 \mu \mathrm{g} / \mathrm{ml})$ and then washed in

A

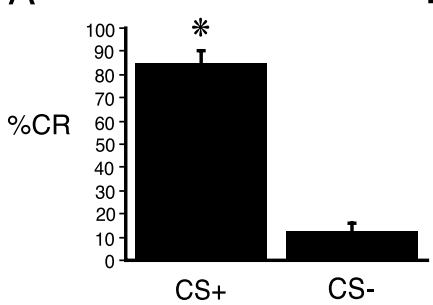

\section{B}

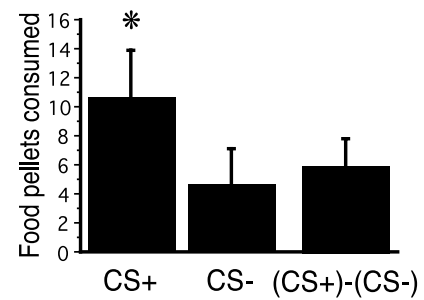

Figure 2. $\quad \boldsymbol{A}$, Conditioning. Acquired CRs (food cup entry) to CS + and $C S-$ on the last day of training. The CRs to $C S+$ and $C S$ - are expressed as the mean percentage of total behavior during the CS presentation. $\boldsymbol{B}$, Food-consumption tests; food consumed by sated rats in the presence of $\mathrm{CS}+$ and in the presence of $\mathrm{CS}$ - . The asterisks refer to statistically reliable differences between CS + and CS - (see Results for details). Error bars represent SEM. mRNA and for FG detection. Then, the tissue was counterstained with

descending concentrations of SSC $(2 \times, 1 \times$, and $0.5 \times$ at room temperature and $0.1 \times$ at $60^{\circ} \mathrm{C}$ ). Tissue was then switched to the Tris buffer system and blocked for $30 \mathrm{~min}$ [blocking agent supplied with PerkinElmer (Wellesley, MA) Tyramide Signal Amplification system; see below]. Slides were then incubated with anti-FG (Chemicon) and antidigoxigenin horseradish peroxidase (HRP) antibody conjugate (Roche Applied Science) overnight at room temperature. The next day, antirabbit antibody conjugated with Alexa Fluor 488 (Molecular Probes, Eugene, OR) was used to detect FG, and cyanine 3 (CY3) substrate kit (NEL704A; PerkinElmer) was used to detect Arc. Slides were treated with $3 \% \mathrm{H}_{2} \mathrm{O}_{2}$ to quench any residual HRP activity, and the fluoresceinlabeled probe that targeted the $3^{\prime}$-UTR of $H 1 a$ was detected with antifluorescein-HRP conjugate (Roche Applied Science) and then a CY5 substrate kit (NEL705A; PerkinElmer). Nuclei were counterstained with DAPI (Vectashield with DAPI, Vector Laboratories).

Image acquisition and analysis. Brain sections processed with combined double-label fluorescence in situ hybridization, immunofluorescence for FG, and counterstain for nuclei, were analyzed with confocal microscopy. Images were acquired on a Zeiss (Oberkochen, Germany) LSM 510 META confocal microscope (laser-scanning system LSM 510; Zeiss) equipped with argon, helium-neon, and diode lasers. Confocal $z$-stacks composed of 1- $\mu$ m-thick optical sections were collected through the regions of interest (BL/BM, CEA, ACB, and omPFC). A typical confocal stack had $\sim 201-\mu \mathrm{m}$-thick optical sections. Stacks were collected at $63 \times$ oil objective. The settings [the pinhole and photomultiplier tube (PMT) gain and contrast] were kept constant for all image stacks acquired from a slide. The settings were optimized for detecting intranuclear signal by adjusting the laser power settings and PMT gain accordingly.

The anatomical borders for the brain areas analyzed were demarcated according to the Swanson (1998) atlas. The nuclear counterstain DAPI was used as one visual guide to demarcate anatomical borders. Adjacent thionin-stained sections were also consulted to confirm anatomical borders. Each brain was systematically examined, and each structure of interest was demarcated throughout its entire rostrocaudal length. z-stacks were collected uniformly throughout the entire extent of the area/nucleus that contained FG-labeled neurons. This yielded $\sim 250-300$ FGlabeled neurons per animal in areas that showed dense FG labeling (such as omPFC and BL/BM) and $\sim 70-100$ FG-labeled neurons per animal in areas that did not have as many FG-labeled neurons (such as CEA and ACB). Using Zeiss LSM ImageBrowser software, confocal z-stacks were analyzed by examining each optical section, which allowed us to ensure that only entire neurons were included in the analysis (i.e., presence of $80 \%$ cell bodies in all $x, y$, and $z$ planes), as described previously (Guzowski and Worley, 2001). The FG-labeled neurons were identified and counted. Then, the nuclei (identified with DAPI) of the FG-labeled neurons were systematically evaluated through $z$-planed sections for IEG mRNA. FG-labeled neurons that contained only Arc intranuclear foci (INF) were classified as $A r c+$, and the FG-labeled neurons that contained only Hla INF were classified as Hlat, and the FG-labeled neurons that contained both $\operatorname{Arc}$
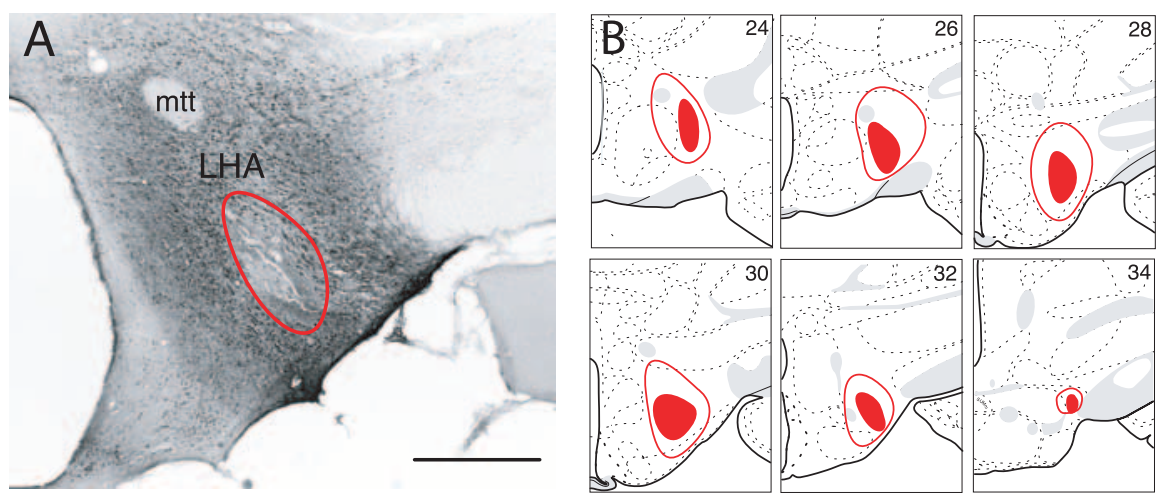
and H1a INF were classified as ArcéHlat. Thus, we observed four types of labeled neurons: nonprojecting neurons (single-labeled neurons; DAPI), neurons that project to the LHA (double-labeled neurons; FG plus DAPI), projecting neurons that are labeled with one IEG (triple-labeled neurons; FG plus $\operatorname{Arc}$ or H1a plus DAPI), and projecting neurons that were labeled with both IEGs (quadruplelabeled neurons; FG plus Arc plus H1a plus DAPI). The focus of the analysis was to determine whether neurons that project to LHA (FG labeled) are activated by tests with CS - or CS + selectively. Thus, the FG-labeled neurons that contained only one of the two IEGs (only Arc INF or only H1a INF) were selective to one of the two tests, whereas the neurons with both $\mathrm{Arc}$ and $\mathrm{Hla}$ INF were considered nonselective.
Figure 3. A, A photomicrograph of the hypothalamus in the immunohistochemically processed tissue after $F G$ injection. The red line encircles the area of the FG deposit within the LHA. Scale bar, $500 \mu \mathrm{m}$. $\boldsymbol{B}$, The extent of the largest (enclosed red area) and smallest (filled red area) FG deposit. Plates were adapted from the atlas of Swanson (1998). mtt, Mammillothalamic tract. 

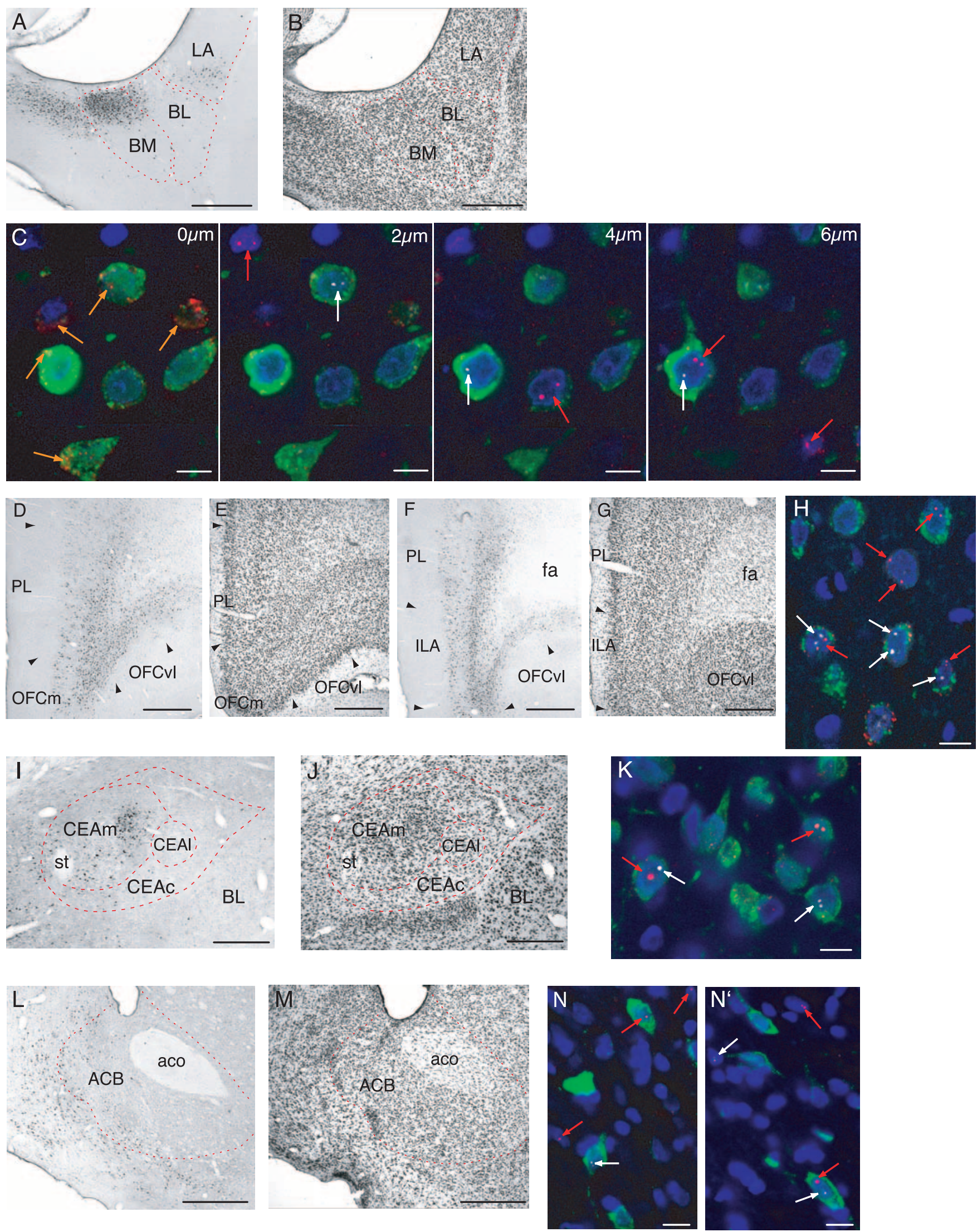

Figure 4. Photomicrographs showing retrogradely labeled neurons (black deposits) within the BL/BM ( $\boldsymbol{A}$, an adjacent thionin-stained section shown in $\boldsymbol{B})$, rostral and caudal omPFC (D, $\boldsymbol{F}$, adjacent thionin-stained sections shown in $\boldsymbol{E}, \boldsymbol{G}),(E A(\boldsymbol{l}$, an adjacent thionin-stained section shown in $\boldsymbol{J})$, and ACB ( $\boldsymbol{L}$, an adjacent thionin-stained section shown in $\boldsymbol{M})$ after $F G$ injection in the $L H A$ (shown in Fig. 3).C, High-power photomicrographs of 1- $\mu \mathrm{m}$-thick optical confocal sections showing a group of neurons in the BM through the z-axis at 0,2,4, and $6 \mu \mathrm{m}$, after combined FG detection with double-label FISH for Arc and H1a mRNA. FG-labeled neurons are visible in green; nuclei counterstained with DAPI are shown in blue; Arc INF are shown in red (red arrow); H1a INF are shown in white (white arrow). Cytoplasmic Arc mRNA, the appearance of which coincides with $H 1$ a INF, is also visible ( $\mu \mathrm{m}$; orange arrow). Four types of stained neurons that were analyzed are as follows: 
The number of FG neurons that also contained IEGs were expressed as a percentage of the total number of FG-labeled neurons counted in a given area. The above analyses were completed on brains from eight rats for all regions, with the exception of omPFC, in which material was available for only seven of the rats in the study. An experimenter, blind to the order of the food-consumption tests with CS+ and CS-, performed all analysis. The order was counterbalanced for tests with CS - and CS+ presentations. The counterbalanced design absorbed any error that would occur because of the order of stimuli (Arc INF staining was induced by the second test, whereas H1a INF staining was induced by the first test), and errors related to processing (Arc riboprobe was always labeled with digoxigenin-UTP that was visualized by CY3, whereas H1a riboprobe was always labeled with fluorescein-UTP that was visualized by CY5; see above). Thus, in one-half of the cases, Arc INF represented tests with $\mathrm{CS}+$ presentation, and H1a INF represented tests with CS- presentations, whereas for the other half of the cases, the opposite was true.

Behavioral observations. Observations were made from the videotapes of the behavioral conditioning sessions by experimenters who were blind to group assignments. The observations were paced by auditory signals (at $1.25 \mathrm{~s}$ intervals) recorded onto the tapes. At each observation, only one behavior was recorded. The primary measure of conditioning, conditioned responses (CRs) to the auditory CSs, was the percentage of time that the rats spent expressing food cup behavior during the CS intervals. Food cup behavior consisted of nose pokes into the recessed food cup, standing motionless in front of the food cup, or short, rapid, horizontal, or vertical head jerks (in the vicinity of the food cup).

Statistics. Behavioral data were analyzed using paired $t$ tests and ANOVA, followed by post hoc tests when appropriate. In all cases, $p<$ 0.05 was considered significant.

\section{Results}

\section{Behavioral testing}

Hungry rats maintained on a scheduled feeding regimen were trained in a pavlovian discrimination task, in which presentations of a tone, CS+, were paired with food delivery, whereas presentations of another auditory stimulus, the CS-, were not paired with food delivery. After rats acquired this discrimination, the occurrence of CRs directed to the food cup during the presentation of the CS + , which predicted food, were significantly elevated compared with CRs during the CS $-\left(t_{(11)}=14.217 ; p<0.0001\right)$ (Fig. 2A). After training, which was conducted in a foodrestricted state, rats were allowed food ad libitum for 2 weeks. Food-consumption tests for potentiated eating were then performed in the sated condition when food was available in the test apparatus in the presence of the CS+ in one test and in the presence of CS - in the other test. The order of the tests with CS+ and CS - was counterbalanced among the rats in the study. As expected from previous work using these procedures, rats ate significantly more food pellets in the presence of CS + compared with tests with CS $-\left(t_{(11)}=3.936 ; p<0.01\right)$ (Fig. $2 B$ ). Those results indicate that augmented eating in sated rats is a result of the previously learned relationship between the predictive cue and food rather than some nonspecific activation by an auditory stimulus, because the paired CS + , but not the unpaired CS-, elevated food consumption.

\section{Combined double-label FISH and retrograde detection of projection neurons activated in food-consumption tests}

To map functional circuitry that mediates CS-potentiated eating, we used combined IEG and tract-tracing methods. We examined the induction of $A r c$ mRNA and H1a mRNA in neurons that project to LHA, which were identified with the retrograde tracer FG.

The focus of the present study was to examine whether pathways that could form a functional AMY-LHA circuit were activated selectively by the $\mathrm{CS}+$ in food-consumption tests. Injection of the retrograde tracer FG into the LHA was used to identify projection neurons to this region. The location of FG injection sites within the LHA is shown in Figure 3. One series of brain sections from each animal was first processed for FG detection alone, and then, once the accuracy of injection into the target region of the LHA was confirmed, an adjacent series of sections was processed with combined double-label FISH (for Arc and H 1 a mRNA) and FG detection. Figure 4 illustrates the FG-labeled neurons in the areas of interest for our analysis. In agreement with previous studies, we found a substantial number of retrogradely labeled neurons in the BL and BM after FG injections into the LHA (Ono et al., 1985; Petrovich et al., 2001). Most FGlabeled neurons in this region were concentrated in the posterior $\mathrm{BM}$ and an adjacent small dorsomedial strip of the posterior $\mathrm{BL}$ (Fig. 4A). As shown in Figure 4, D and $F$, a large number of FG-labeled neurons were located along the medial wall of the prefrontal cortex, within the prelimbic and infralimbic areas extending into the medial and ventrolateral orbitofrontal cortical areas. Projections from these regions of prefrontal cortex to the lateral hypothalamus have been described previously (Kita and Oomura, 1982; Sesack et al., 1989; Hurley et al., 1991; Risold et al., 1997; Floyd et al., 2001). These prefrontal regions are also known to receive input from BL, BM, and LA (Krettek and Price, 1977; Kita and Kitai, 1990; McDonald, 1991; Swanson and Petrovich, 1998). The CEA (Fig. 4I) and ACB (Fig. 4L), which are targets of BL, BM, and LA innervation (Swanson and Petrovich, 1998), had FG-labeled neurons; in the former case, the projection neurons to LHA were concentrated within a distinct zone of the medial CEA [a region that corresponds to the "intermediate subdivision" described by McDonald (1982)] and, in the latter case, in the caudal shell region of the ACB. This distribution of FG labeling is consistent with previous anatomical tracing of projections from those nuclei to LHA (Krettek and Price, 1978; Kita and Oomura, 1982; Zahm et al., 1999).

Using the retrograde map of labeled projection neurons as a guide, we generated confocal z-stacks through each region of interest for analysis. In that material, we identified FG-positive neurons and then determined whether each FG-positive neuron also contained IEG labeling, as illustrated in Figure 4. The effector IEGs Arc and Hla have coincidence expression in the same neurons with temporally offset appearance/disappearance that can be detected in the nucleus as INF staining (Vazdarjanova et al., 2002; Vazdarjanova and Guzowski, 2004). After neuronal activation, INF signal for Arc and $H 1 a$ peaked at 2-10 and 25-35 min, respectively. Thus, neurons that were labeled with only one of the 
Table 1. The proportion of total identified neurons within BL/BM, omPFC, $A C B$, and CEA, which project to the LHA (FG-labeled), that were labeled with IEG markers in response to food consumption tests

\begin{tabular}{lrrll}
\hline Region & \multicolumn{1}{c}{ CS $+(\%)$} & \multicolumn{1}{c}{ CS $-(\%)$} & CS + and CS $-(\%)$ & FG only (\%) \\
\hline BL/BM & $19.8 \pm 2.9$ & $8.0 \pm 2.2$ & $40.2 \pm 2.0$ & $32.0 \pm 4.4$ \\
omPFC & $19.0 \pm 1.7$ & $7.3 \pm 0.5$ & $43.9 \pm 3.2$ & $29.8 \pm 3.4$ \\
ACB & $9.2 \pm 2.6$ & $8.2 \pm 3.4$ & $25.0 \pm 4.0$ & $57.6 \pm 5.8$ \\
CEA & $5.7 \pm 2.2$ & $13.3 \pm 3.4$ & $31.9 \pm 3.7$ & $49.1 \pm 6.9$ \\
\hline
\end{tabular}

The first two columns show the percentage (mean \pm SEM) of total projecting neurons in which IEG markers were induced selectively by the tests with $C S+$ presentations $(C S+)$ or by the tests with $C S-$ presentations $(C S-)$. The third column shows the percentage (mean \pm SEM) of total projecting neurons in which the IEG markers were induced nonselectively by both tests (CS + and $C S-$ ). The fourth column shows the percentage (mean \pm SEM) of total projecting neurons that did not contain the IEG markers.

two IEGs reflected selective induction in one of the two foodconsumption tests; $\mathrm{H} 1 \mathrm{a}$ INF labeling reflected IEG induction related to the first of the two tests, whereas Arc INF reflected IEG induction of the second of the two tests (Fig. 1). Because the consumption tests were counterbalanced for CS + and CS - , presentations of those events were equally represented by the different IEGs across the rats in the experiment, such that, in one-half of the cases, induction of mRNA for $A r c$ was associated with tests of $\mathrm{CS}+$, and induction of mRNA for $\mathrm{Hla}$ was associated with tests of CS - , and, in the other half of the cases, the opposite was true. The overall Arc or Hla mRNA expression in response to the consumption tests was not dependent on the order of the tests in the results that follow (no significant effect of test order in preliminary ANOVAs). In addition to neurons that showed selective induction of IEG markers in response to one of the two tests, we identified projecting neurons that were nonselective: FG-labeled neurons that contained both IEG markers (Fig. 4). Such nonselective activity within afferents to LHA could reflect nonassociative responsiveness to task-related events such as placement in the chambers, the presence of food, or eating itself, which occurred to some degree in both sessions. We also found projecting neurons that did not contain IEG markers. The proportion of total identified neurons that project to the LHA that were labeled with IEG markers in response to food-consumption tests is shown in Table 1.

Confocal analysis of the BL/BM region that contained neurons projecting to the LHA (FG-positive neurons) revealed that the population of FG- and IEG- (Arc or H1a INF) labeled neurons was always larger in response to tests with $\mathrm{CS}+$ compared with tests with CS - (Fig. 5$)\left(t_{(7)}=3.124 ; p<0.05\right)$, consistent with the acquired power of the CS + to augment food consumption (Fig. 2B). The analysis of prefrontal cortical regions, collectively referred to here as orbitomedial regions (omPFC) also revealed a preponderance of neurons that project to the LHA, indicating activation by $\mathrm{CS}+$ relative to $\mathrm{CS}-($ Fig. 5$)\left(t_{(6)}=\right.$ 7.615; $p<0.001$ ). Different omPFC regions analyzed here (prelimbic, infralimbic, medial, and ventrolateral orbitofrontal areas) are shown together because the percentages of FG neurons that were activated by $\mathrm{CS}+$ or $\mathrm{CS}-$ were similar throughout those areas. Additionally, proportions of activated neurons in those regions did not merely correlate with the amount eaten in the food-consumption tests with $\mathrm{CS}+$; $r$ values for $\mathrm{BL} / \mathrm{BM}$ and omPFC were 0.085 and 0.185 , respectively.

In contrast to the results obtained in the omPFC and BL/BM, we did not find that a larger percentage of the FG-labeled neurons within the CEA or ACB were selectively activated by tests with $\mathrm{CS}+$ (Fig. 5). Instead, we found approximately equal numbers of ACB neurons that are double labeled with FG and IEG (Arc or $H 1 a$ INF) after the tests with CS + and tests with $\mathrm{CS}-\left(t_{(7)}=\right.$

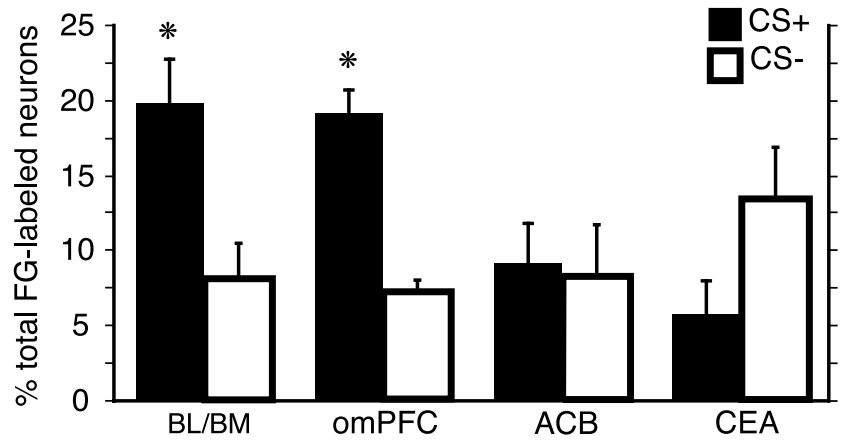

Figure 5. The percentage of total FG-labeled neurons that were labeled with one of the IEG markers (Arc INF or H1a INF) after food-consumption tests (Fig. 2B) in the presence of CS+ compared with tests with $C S-$ in the BL/BM, omPFC, $A C B$, and CEA. The asterisks refer to statistically reliable differences between $C S+$ and $C S-$ (for details, see Results). Error bars represent SEM.

$0.255 ; p=0.806)$. Interestingly, within the CEA, we found that the percentage of the total projecting neurons that are also labeled with one IEG ( $\mathrm{Arc}$ or H1a INF) in response to the tests with CSwere greater compared with the tests with CS + (Fig. 5), although this effect did not reach significance $\left(t_{(7)}=-2.183 ; p=0.065\right)$.

\section{Discussion}

This study demonstrates that direct pathways from the BL/BM and omPFC to the LHA form a functional network for control of eating by learned appetitive cues. Greater food consumption in the presence of the CS + relative to the CS - was dependent on the training histories of those cues in a behavioral model that drives food consumption independent of energy requirements. The anatomical methods we used allowed the detection of projection neurons that were selectively activated by the reinforced $\mathrm{CS}+$.

Projection neurons selectively activated by the cue that potentiates feeding $(\mathrm{CS}+)$ were heavily represented in BL/BM and omPFC regions of cortex. That observation extends our previous findings on the importance of this region of the amygdala and pathways connecting it with the LHA for the control of feeding by cues that gain motivational strength through learning. Thus, conditioned potentiation of feeding, using behavioral procedures similar to those used in the current neuroanatomical analysis, is abolished by neurotoxic lesions that encompass BL/BM/LA or disconnection of that region of the amygdala and LHA. Notably, several other routes for access to the LHA from the BL/BM/LA, via $\mathrm{CEA}$ and $\mathrm{ACB}$, did not provide evidence for mediating the effects of motivationally powerful cues on food consumption. Although a variety of evidence has implicated those regions of the forebrain in aspects of motivational control connected with feeding (Cardinal et al., 2002; Berridge, 2003; Phillips et al., 2003; Kelley, 2004) projection neurons to LHA in those regions were not preferentially activated by the CS + .

The small proportion of CEA projection neurons that were activated by the CS + is in agreement with previous behavioral results showing that CEA is not needed for conditioned potentiation of feeding (Holland et al., 2002). In the current analysis, we actually found a larger percentage of CEA neurons projecting to the LHA that were activated in tests with the $\mathrm{CS}-$ relative to $\mathrm{CS}+$, although that comparison was not statistically significant. Because the CEA is critical for a number of behaviors that rely on associative learning, including its well known role in aversive conditioning (LeDoux, 2000; Davis et al., 2003; Fanselow and Poulos, 2005), it is tempting to speculate that somewhat greater 
activation of CEA neurons by the CS - might be attributable to aversive properties acquired by that cue during training, when it was presented to hungry rats in the absence of food. However, the absence of controls for the identification of such a CS - function, such as a random control group (Rescorla, 1967), precludes any such conclusion in this study.

The current study provides the first evidence for learningdependent activation of pathways from the forebrain directly targeting the hypothalamic system that controls feeding. The amygdala (BL/BM) and omPFC network revealed in this investigation is especially interesting in light of findings from functional imaging studies that have identified these forebrain structures with the motivational control of appetite in humans. Activations of the amygdala and "medial orbitofrontal cortical area" are seen when hungry humans view food items relative to nonfood items, and greater activations are related to the higher incentive value among the food items that are viewed (Arana et al., 2003; Hinton et al., 2004). Moreover, activation of the amygdala has been specifically noted in a number of experimental settings. Cues for foods that retain incentive value evoke greater amygdala activation relative to cues for food items that were recently consumed to satiation (Gottfried et al., 2003). At the same time, exposure to highly preferred food items on a menu will recruit activity in this region regardless of whether neuroimaging is performed during fasting or after participants have eaten (Hinton et al., 2004). The medial orbital area identified in human neuroimaging studies using food-related stimuli (Arana et al., 2003; Hinton et al., 2004; DelParigi et al., 2005) is difficult to relate to those omPFC neurons activated in the current investigation, given uncertainties about the homology of prefrontal cortical areas in different species. However, connectional anatomy and functional/behavioral parallels have helped to define a close correspondence for circuits in the medial and orbital regions across rodents, monkeys, and humans (Öngür and Price, 2000; Schoenbaum et al., 2002; Holland and Gallagher, 2004). Thus, the results reported here suggest that such regions of forebrain activation may gain direct access to circuitry for the control of food consumption in humans, providing routes for the motivational control of appetite and the behavioral regulation of eating under extrinsic influences. Given its important role in goal-directed behavior (O'Doherty, 2004), prefrontal pathways from omPFC, in particular, could play a pivotal function in regulating the impulse to eat in response to appetitive cues with high incentive value.

\section{References}

Arana FS, Parkinson JA, Hinton EC, Holland AJ, Owen AM, Roberts AC (2003) Dissociable contribution of the human amygdala and orbitofrontal cortex to incentive motivation and goal selection. J Neurosci 23:9632-9638.

Berridge KC (2003) Parsing reward. Trends Neurosci 26:507-513.

Berthoud H-R (2002) Multiple neural systems controlling food intake and body weight. Neurosci Biobehav Rev 26:393-428.

Booth DA (1989) Mood- and nutrient-conditioned appetities. Cultural and physiological bases for eating disorders. Ann NY Acad Sci 575:122-135.

Brakeman PR, Lanahan AA, O’Brien R, Roche K, Barnes CA, Huganir RL, Worley PF (1997) Homer: a protein that selectively binds glutamate receptors. Nature 386:284-288.

Cardinal RN, Parkinson JA, Hall J, Everitt BJ (2002) Emotion and motivation: the role of the amygdala, ventral striatum, and prefrontal cortex. Neurosci Biobehav Rev 26:321-352.

Davis M, Walker DL, Myers KM (2003) Role of the amygdala in fear extinction measured with potentiated startle. Ann NY Acad Sci 985:218-232.

DeFalco J, Tomishima M, Liu H, Zhao C, Cai XL, Marth JD, Enquist L, Friedman JM (2001) Virus-assisted mapping of neural inputs to a feeding center in the hypothalamus. Science 291:2608-2613.

DelParigi A, Chen K, Salbe AD, Reiman EM, Tataranni A (2005) Sensory experience of food and obesity: a positron emission tomography study of the brain regions affected by tasting a liquid meal after a prolonged fast. NeuroImage 24:436-443.

Fanselow MS, Poulos AM (2005) The neuroscience of mammalian associative learning. Annu Rev Psychol 56:207-234.

Floyd NS, Price JL, Ferry AT, Keay KA, Bandler R (2001) Orbitomedial prefrontal cortical projections to hypothalamus in the rat. J Comp Neurol 432:307-328.

Gottfried JA, O’Doherty J, Dolan RJ (2003) Encoding predictive reward value in human amygdala and orbitofrontal cortex. Science 301:1104-1107.

Grill HJ, Kaplan JM (2002) The neuroanatomical axis for control of energy balance. Front Neuroendocrinol 23:2-40.

Guzowski JF, Worley PF (2001) Cellular compartment analysis of temporal activity by fluorescent in situ hybridization (catFISH). In: Current protocols in neuroscience (Taylor GP, ed), pp 1-16. New York: Wiley.

Hinton EC, Parkinson JA, Holland AJ, Arana FS, Roberts AC, Owen AM (2004) Neural contribution to the motivational control of appetite in humans. Eur J Neurosci 20:1411-1418.

Holland PC, Gallagher M (2004) Amygdala-frontal interactions and reward expectancy. Curr Opin Neurobiol 14:148-155.

Holland PC, Petrovich GD, Gallagher M (2002) The effects of amygdala lesions on conditioned stimulus-potentiated eating in rats. Physiol Behav 76:117-129.

Hurley KM, Herbert H, Moga MM, Saper CB (1991) Efferent projections of the infralimbic cortex of the rat. J Comp Neurol 308:249-276.

Kelley AE (2004) Ventral striatal control of appetitive motivation: role of ingestive behavior and reward-related learning. Neurosci Biobehav Rev 27:765-776.

Killgore WDS, Young AD, Femia LA, Bogorodzki P, Rogowska J, YurgelunTodd DA (2003) Cortical and limbic activation during viewing of highversus low-calorie foods. NeuroImage 19:1381-1394.

Kita H, Kitai ST (1990) Amygdaloid projections to the frontal cortex and the striatum in the rat. J Comp Neurol 298:40-49.

Kita H, Oomura Y (1982) An HRP study of the afferent connections to rat lateral hypothalamic region. Brain Res Bull 8:63-71.

Krettek JE, Price JL (1977) Projections from the amygdaloid complex to the cerebral cortex and thalamus in the rat and cat. J Comp Neurol 172:687-722.

Krettek JE, Price JL (1978) Amygdaloid projections to subcortical structures within the basal forebrain and brainstem in the rat and cat. J Comp Neurol 178:225-254.

Kringelbach ML, O’Doherty J, Andrews C (2003) Activation of human orbitofrontal cortex to a liquid food stimulus is correlated with its subjective pleasantness. Cereb Cortex 13:1064-1071.

LaBar KS, Gitelman DR, Parrish TB, Kim YH, Nobre AC, Mesulam MM (2001) Hunger selectively modulates corticolimbic activation to food stimuli in humans. Behav Neurosci 115:493-500.

LeDoux JE (2000) Emotion circuits in the brain. Annu Rev Neurosci 23:155-184.

Link W, Konietzko U, Kauselmann G, Krug M, Schwanke B, Frey U, Kuhl D (1995) Somatodendritic expression of an immediate early gene is regulated by synaptic activity. Proc Natl Acad Sci USA 92:5734-5738.

Lyford GL, Yamagata K, Kaufmann WE, Barnes CA, Sanders LK, Copeland NG, Gilbert DI, Jenkins NA, Lanahan AA, Worley PF (1995) Arc, a growth factor and activity-regulated gene, encodes a novel cytoskeletonassociated protein that is enriched in neuronal dendrites. Neuron 14:433-445.

McDonald AJ (1982) Cytoarchitecture of the central amygdaloid nucleus of the rat. J Comp Neurol 208:401-418.

McDonald AJ (1991) Organization of amygdaloid projections to the prefrontal cortex and associated striatum in the rat. Neuroscience 44:1-14.

Moran T (2004) Gut peptides in the control of food intake: 30 years of ideas. Physiol Behav 82:175-180.

O'Doherty JP (2004) Reward representations and reward-related learning in the human brain: insights from neuroimaging. Curr Opin Neurobiol 14:769-776.

Öngür D, Price JL (2000) The organization of networks within the orbital and medial prefrontal cortex of rats monkeys and humans. Cereb Cortex 10:206-219.

Ono T, Luiten PGM, Nishijo H, Fukuda M, Nishino H (1985) Topographic 
organization of projections from the amygdala to the hypothalamus of the rat. Neurosci Res 2:221-239.

Petrovich GD, Canteras NS, Swanson LW (2001) Combinatorial amygdalar inputs to hippocampal domains and hypothalamic behavior systems. Brain Res Rev 38:247-289.

Petrovich GD, Setlow B, Holland PC, Gallagher M (2002) Amygdalohypothalamic circuit allows learned cues to override satiety and promote eating. J Neurosci 22:8748-8753.

Phillips AG, Ahn S, Howland JG (2003) Amygdalar control of the mesocorticolimbic dopamine system: parallel pathways to motivated behavior. Neurosci Biobehav Rev 27:543-554.

Rescorla RA (1967) Pavlovian conditioning and its proper control procedures. Psychol Rev 74:71-80.

Risold PY, Thompson RH, Swanson LW (1997) The structural organization of connections between hypothalamus and cerebral cortex. Brain Res Rev 24:197-254.

Rodin J (1981) Current status of the internal-external hypothesis for obesity. Am Psychol 36:361-372.

Saper CB, Chou TC, Elmquist JK (2002) The need to feed: homeostatic and hedonic control of eating. Neuron 36:199-211.

Schoenbaum G, Setlow B, Gallagher M (2002) Orbitofrontal cortex: modeling prefrontal function in rats. In: The neuropsychology of memory (Squire L, Schacter D, eds). New York: Guilford.

Schwartz MW, Woods SC, Porte DJ, Seeley RJ, Baskin DG (2000) Central nervous system control of food intake. Nature 4040:661-671.

Sesack SR, Deutch AY, Roth RH, Bunney BS (1989) Topographical organization of the efferent projections of the medial prefrontal ortex in the rat: an anterograde tract-tracing study with Phaseolus vulgaris leucoagglutinin. J Comp Neurol 290:213-242.
Simmons DM, Arriza JL, Swanson LW (1989) A complete protocol of in situ hybridization of messenger RNAs in brain and other tissues with radiolabeled single-stranded RNA probess. J Histotechnol 12:169-181.

Stroebele N, De Castro JM (2004) Effect of ambience on food intake and food choice. Nutrition 20:821-838.

Swanson LW (1998) Brain maps: structure of the rat brain, Ed 2. Amsterdam: Elsevier.

Swanson LW (2000) Cerebral hemisphere regulation of motivated behavior. Brain Res 886:113-164.

Swanson LW, Petrovich GD (1998) What is the amygdala? Trends Neurosci 21:323-331.

Tataranni PA, Gautier J-F, Chen K, Uecker A, Bandy D, Salbe AD, Pratley RE, Lawson M, Reiman EM, Ravussin E (1999) Neuroanatomical correlates of hunger and satiation in humans using positron emission tomography. Proc Natl Acad Sci USA 96:4569-4574.

Vazdarjanova A, Guzowski JF (2004) Differences in hippocampal neuronal population responses to modificiations of an environmental context: evidence for distinct, yet complementary, functions of CA3 and CA1 ensembles. J Neurosci 24:6489-6496.

Vazdarjanova A, McNaughton BL, Barnes CA, Worley PA, Guzowski JF (2002) Experience-dependent coincident expression of the effector immediate-early genes Arc and Homer $1 a$ in hippocampal and neocortical neuronal networks. J Neurosci 22:10067-10071.

Weingarten HP (1983) Conditioned cues elicit feeding in sated rats: a role for learning in meal initiation. Science 220:431-433.

Zahm DS, Jensen SL, Williams ES, Martin III JR (1999) Direct comparison of projections from the central amygdaloid region and nucleus accumbens shell. Eur J Neurosci 11:1119-1126. 